\title{
Condições de saúde e risco de internação hospitalar em idosas praticantes de hidroginástica
}

\author{
Health conditions and admission hospital risk in elderly women
} practicing waters aerobics

\author{
Emanuelly Casal Bortoluzzi* \\ Temístocles Vicente Barros** \\ Caroline Brand ${ }^{* * *}$ \\ Patrícia Fagundes Soares**** \\ Maria Amélia Roth****
}

\begin{abstract}
Resumo
O envelhecimento populacional traz uma nova realidade ao sistema de saúde, visto que estes são mais propensas a doenças crônicas, as quais demandam um alto custo. Objetivou-se analisar a prevalência de dispneia e fadiga e sua correlação com medidas antropométricas, além de condições de risco de internação hospitalar em idosas praticantes de hidroginástica. Trata-se de estudo transversal, quantitativo, com 94 idosas praticantes de hidroginástica. Realizouse coleta de dados por meio de questionários de avaliação de risco de internação hospitalar e função pulmonar e dispneia, além de medidas antropométricas. Os resultados apontaram valor da média do IMC, CC e RCQ acima do ideal (28,6/ 93,6/ 0,87 respectivamente). A autopercepção de saúde foi positiva para 74,3\% das idosas e cerca de 90\% não tiveram internações nos últimos doze meses. Ainda, há correlação negativa, significativa, entre o tempo de prática de hidroginástica e fadiga. Portanto, percebe-se os efeitos positivos da pratica de hidroginástica, visto que mesmo com resultados antropométricos alterados, a grande maioria das idosas relataram boas condições de saúde, baixo número de consultas para tratamento e internações hospitalares. Quanto a dispneia e fadiga, percentual em torno de 5\% referiram possuir dispneia e fadiga grave/muito grave, os quais necessitam de cuidados adequados.
\end{abstract}

Palavras-chave: Idoso. Atividade motora. Dispneia. Exercício. Hospitalização.

\begin{abstract}
The aging brings a new reality to the health system, since they are more prone to chronic diseases, which demand a high cost. This study aimed to analyze the prevalence of dyspnea and fatigue and its correlation with anthropometric measurements, and hospitalization risks in elderly women engaged in water aerobics. This cross-sectional, quantitative study with 94 elderly women engaged in water aerobics. Held data collection through risk assessment questionnaires hospitalization and pulmonary function and dyspnea, and anthropometric measurements. The results showed average value of BMI, WC and WHR above the ideal (28.6 / 93.6 / 0.87 respectively). The self-rated health was positive for $74.3 \%$ of the women and about $90 \%$ had no hospitalizations in the last twelve months. Still, there are significant negative correlation between the water aerobics practice time and fatigue. Therefore, we can see the positive effects of practicing gymnastics since even with altered anthropometric results, the vast majority of older reported good health, low number of visits for treatment and hospitalization. As dyspnea and fatigue, percentage around 5\% reported having dyspnea and severe/very severe fatigue, which require appropriate care.
\end{abstract}

Keywords: Aged. Motor Activity. Dyspnea. Exercise. Hospitalization.

DOI: $10.15343 / 0104-7809.20164003293301$

\footnotetext{
* Universidade de Passo Fundo (UPF). Passo Fundo - RS, Brasil. E-mail: manu_casal@hotmail.com **Universidade de Santa Maria (UFSM). Santa Maria - RS, Brasil. E-mail: netobarro.ef@gmail.com.

***Universidade Federal do Rio Grande do Sul (UFRGS). Porto Alegre - RS, Brasil. E-mail: carolbrand@hotmail.com.

**** Universidade de Santa Maria (UFSM). Santa Maria - RS, Brasil. E-mail: patifagundessoares@hotmail.com

***** Universidade de Santa Maria (UFSM). Santa Maria - RS, Brasil. E-mail: necaroth@gmail.com

Os autores declaram não haver conflitos de interesse.
} 


\section{INTRODUÇÃO}

O fenômeno atual do envelhecimento é uma realidade social e um grande desafio devido à mudança na pirâmide etária, a qual apresenta perspectivas de estrutura cilíndrica nas próximas décadas. No Brasil, são considerados idosos pessoas com idade igual ou superior a 60 anos, faixa etária que vem crescendo rapidamente, em especial, os idosos com 80 anos e mais, tem o maior índice de crescimento atualmente, representam cerca de $1,5 \%$ da população brasileira $^{1}$ (IBGE, 2013).

Tendo como foco o sistema de saúde, podese destacar as mudanças nas características das principais causas de morbimortalidades no mundo, passando de doenças infecciosas para doenças não transmissíveis, concentrando-se em quatro doenças: cardiovasculares, diabetes, câncer e doenças respiratórias crônicas ${ }^{3}$. No Brasil, elas correspondem a principal causa de morte $^{2}$, o que acomete de igual forma a maioria dos países do mundo, devido ao rápido crescimento da economia nacional e as mudanças no meio ambiente e estilo de vida ${ }^{2,3}$.

A nova característica das doenças faz necessário um novo modelo de saúde, no qual a promoção e proteção à saúde seja efetiva, e não apenas a recuperação através de tratamentos agudos. Em geral, as doenças dos idosos perduram por vários anos e exigem acompanhamento médico e de equipes multidisciplinares permanentes, além de intervenções contínuas ${ }^{4}$.

Com o envelhecimento populacional há um aumento na utilização dos serviços de saúde e elevado índice de permanência e reinternação hospitalar ${ }^{4}$ o que acarreta altos custos ao sistema único de saúde (SUS) e ao indivíduo, além de seu sofrimento e de sua família ${ }^{2}$. Doenças do sistema circulatório e respiratório estão entre as mais frequentes causas de internação hospital em ambos os sexos ${ }^{4}$.

A asma, a rinite alérgica e a doença pulmonar obstrutiva crônica (DPOC), são as doenças respiratórias crônicas (DRC) mais comuns ${ }^{5}$, bem como a insuficiência cardíaca é uma das mais importantes doenças cardiovasculares. As quais apresentam como alguns de seus principais sintomas, dispneia e fadiga, contudo por serem considerados efeitos naturais do envelhecimento, muitas vezes, estes indicadores de suma relevância, não são tratados com a devida importância ${ }^{6}$.

Com o envelhecimento ocorrem importantes mudanças cardiorrespiratórias, tais como, decréscimo de até $75 \%$ da capacidade vital entre os 20 e os 70 anos de idade e aumento da capacidade residual funcional, assim os idosos necessitam respirar maiores volumes de ar do que adultos e jovens ${ }^{7}$. Nesse sentido, as perspectivas contemporâneas de cuidados ao envelhecimento humano, incluem as práticas de educação física agregando conhecimento e possibilidades deexercícios físicos para os idosos.

A hidroginástica surge como possibilidade de exercícios globais que proporcionam ganhos musculares e cardiorrespiratórios, agindo sobre os aspectos físicos, psicológicos e sociais ${ }^{8}$, em um ambiente agradável e terapêutico.

Devido as propriedades físicas da água a hidroginástica tem sido praticada com frequência em programas de exercício para a terceira idade $^{10}$. A pressão hidrostática é uma das propriedades da água, a qual possibilita ganhos importantes no sistema respiratório, pois o mesmo sofre alterações devido à densidade da água, 775 vezes maior que a do ar, podendo exercer uma pressão sobre a caixa torácica e o abdômen exigindo um grande esforço na fase inspiratória e uma maior facilidade na expiração, favorecendo o fortalecimento da musculatura respiratória9.

Tendo em vista o fenômeno do envelhecimento populacional e o impacto das doenças cardiorrespiratórias na vida dos indivíduos e no sistema de saúde, este estudo teve como objetivo analisar condições de saúde cardiorrespiratória e risco de internação hospitalar em idosas praticantes de hidroginástica.

\section{MATERIAIS E MÉTODOS}

Trata-se de um estudo de corte transversal, de abordagem quantitativa, com amostragem por conveniência, realizado com idosas 
participantes projeto de Hidroginástica para a Terceira Idade do Centro de Educação Física e Desportos (CEFD) na Universidade Federal de Santa Maria (UFSM). O grupo de estudo foi de 94 idosas, com idades entre 60 e 84 anos,

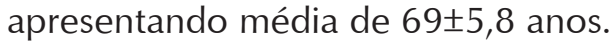

Como critérios de inclusão as mulheres deveriam ter no mínimo 60 anos e serem praticantes de hidroginástica a há pelo menos seis meses, no mínimo duas vezes por semana. As aulas de hidroginástica tinham a duração de 50 minutos (min.), apresentando $10 \mathrm{~min}$. de aquecimento e $30 \mathrm{~min}$. de exercícios na seguinte ordem: leve, moderado, forte, moderado e leve, seguido por $10 \mathrm{~min}$. de volta à calma. $\mathrm{O}$ aquecimento por vezes era uma atividade recreativa dentro da piscina.

Para a realização dos procedimentos as idosas foram conduzidas individualmente à uma sala onde eram habitualmente realizados os exames médicos. As entrevistas foram realizadas no período da manhã, antes das respectivas aulas de hidroginástica ocorridas de segundas-feiras a sábados.

Para a obtenção dos dados, foi aplicado o seguinte protocolo, contemplando os seguintes itens:

- Adaptação da versão em português do questionário Pulmonary Functional and Dyspnea ${ }^{11}, \mathrm{o}$ qual foi adaptado para investigar a presença de fadiga e dispneia no dia da entrevista, no dia-adia e em atividade da vida diária. Para melhor entendimento foram utilizados os termos falta de ar para dispneia e cansaço muito grande para fadiga;

- O Questionário de avaliação de risco de internação hospitalar ${ }^{12}$, composto por questões referentes a autopercepção de saúde, consultas para tratamento, internação, doenças cardíacas, diabetes e rede de apoio.

- Foram coletadas as seguintes medidas antropométricas: massa corporal (balança G.Tech Glass 7 FW) e estatura (estadiômetro fixo, Cardiomed-Brasil), através dos quais foi definido o índice de massa corporal (IMC) obtida pela divisão do peso corporal total $(\mathrm{Kg})$, pela estatura (m). As circunferências da cintura (CC) e quadril (CQ) (fita métrica BIOTEK, $150 \mathrm{~cm}$ ) foram verificadas para definir a relação cintura-quadril (RCQ), obtidas pela divisão dos perímetros da cintura $(\mathrm{cm})$ e do quadril (cm).

Para os valores de IMC foi adotada a classificação adaptada da OMS, utilizada nas Diretrizes Brasileiras de Obesidade (ABESO), assim como os valores de CC. Para RCQ foi aceito $\leq 0,8 \mathrm{~cm}^{13,14}$.

Os questionários foram aplicados no formato de entrevista devido as dificuldades visuais e de leitura, e desejo das entrevistadas percebido no projeto piloto, sendo assim os entrevistadores, previamente treinados, foram orientados a manter esta prática sem interferir nas respostas. Realizou-se análise descritiva e inferencial. Para análise descritiva de variáveis categóricas utilizou-se frequência absoluta e relativa, e para variáveis numéricas utilizou-se média e desviopadrão. Para análise inferencial, os dados foram analisados pelo coeficiente de correlação de Pearson, o nível de significância aceito foi de $p<$ 0,05. O pacote estatístico utilizado foi SAS 9.0.

Antes de serem aplicados os questionários de avaliação, todas as idosas assinaram o Termo de Consentimento Livre e Esclarecido. O presente estudo foi aprovado pelo Comitê de Ética e Pesquisa da UFSM, sob parecer $n^{\circ}$ 929.668.

\section{RESULTADOS}

Com relação as características antropométricas, o valor da média do IMC aponta para pré-obesidade, da mesma forma a média dos valores de CC e RCQ encontram-se acima do ideal (Tabela 1).

Quanto as questões referentes ao questionário de risco de internação hospitalar, destaca-se que apenas $2,1 \%$ das idosas considera sua saúde ruim e cerca de $90 \%$ das idosas não tiveram internações hospitalares nos últimos doze meses (Tabela 2).

Quanto à presença de dispneia, 28,7\% relataram já ter sentido falta de ar em algum momento da vida e 74,4\% disseram já ter sentido cansaço muito grande. Em relação à presença de dispneia e fadiga em atividades diárias, as principais dificuldades foram encontradas em 
subir alguns degraus e em subidas (Tabela 3).

Na tabela 4 serão apresentados os resultados da correlação entre medidas antropométricas, dispneia, fadiga e tempo de prática da hidroginástica. Foram encontradas correlações significativas entre dispneia e IMC (correlação positiva e fraca), dispneia nas atividades diárias e massa corporal e IMC (ambas positivas e fracas), fadiga e massa corporal e IMC (ambas positivas e fracas) e fadiga nas atividades diárias e massa corporal e IMC (ambas positivas e fracas). Quanto ao tempo de prática de hidroginástica esta é correlacionada a fadiga (negativa e fraca).

Tabela 1 - Caracterização dos dados antropométricos, por meio de média \pm DP, Santa Maria, RS, 2014.

\begin{tabular}{|c|c|c|}
\hline Variáveis & Médias(n=94) & DP \\
\hline Massa Corporal (Kg) & 71 & 12,7 \\
\hline Estatura (m) & 1,57 & 0,06 \\
\hline $\mathrm{IMC}\left(\mathrm{Kg} / \mathrm{m}^{2}\right)$ & 28,6 & 4,5 \\
\hline $\mathrm{CC}(\mathrm{cm})$ & 93,6 & 11,2 \\
\hline $\mathrm{CQ}(\mathrm{cm})$ & 106,4 & 9,3 \\
\hline RCQ $(\mathrm{cm})$ & 0,87 & 0,06 \\
\hline
\end{tabular}

DP: desvio padrão; IMC: índice de massa corporal; CC: circunferência da cintura; CQ: circunferência do quadril; RCQ: relação cintura-quadril.

Tabela 2 - Características de saúde e de internação hospitalar em termos numéricos e percentuais, Santa Maria, RS, 2014.

\begin{tabular}{lcc}
\hline \multicolumn{1}{c}{ Variáveis } & $\mathbf{n}$ & $\%$ \\
\hline Autopercepção de saúde & 13 & 13.8 \\
Excelente & 15 & 15.9 \\
Muito boa & 42 & 44.6 \\
Boa & 22 & 23.4 \\
Média & 2 & 2.1 \\
Ruim & & \\
\hline Internações nos últimos $\mathbf{1 2}$ meses & 84 & 89.3 \\
\hline Nenhuma vez & 8 & 8.5 \\
Uma vez & 1 & 1.1 \\
Duas ou três vezes & 1 & 1.1 \\
Mais do que três vezes & & 26.6 \\
\hline Consultas para tratamentos nos últimos $\mathbf{1 2}$ meses & 25 & 26.6 \\
\hline Nenhuma vez & 25 & 25.5 \\
\hline Uma vez & 24 & \\
Duas ou três vezes & & \\
\hline
\end{tabular}


...continuação - Tabela 2

\begin{tabular}{llc}
\hline Quatro a seis vezes & 10 & 10.6 \\
Mais de seis vezes & 10 & 10.6 \\
\hline Possui Diabetes Mellitus & & 17.0 \\
\hline Sim & 16 & 82.9 \\
Não & 78 & 14.8 \\
\hline Possui doença cardíaca & 14 & 85.1 \\
\hline Sim & 80 & 87.2 \\
Não & & 12.7 \\
\hline Presença de amigo, familiar ou vizinho que pudesse cuidá- & 82 & 12 \\
la por alguns dias caso necessário & & \\
\hline Sim & & \\
Não & & \\
\hline
\end{tabular}

Tabela 3 - Descrição em termos percentuais das respostas ao questionário referente a presença de dispneia e fadiga, Santa Maria, RS, 2014.

\begin{tabular}{lccccccccccc}
\hline & \multicolumn{4}{c}{ Dispneia (\%) } & \multicolumn{7}{c}{ Fadiga (\%) } \\
\hline & N & L & M & G & MG & N & L & M & G & MG \\
\hline Maioria dos dias & 88,3 & 5,3 & 5,3 & 1,1 & - & 61,7 & 21,2 & 12,7 & 3,2 & 1,1 \\
Hoje* & 96,8 & 1,1 & 1,1 & 1,1 & - & 89,3 & 4,2 & 3,2 & 1,1 & 1,1 \\
Maioria das ativ. & 94,6 & 1,1 & 3,2 & 1,1 & - & 61,7 & 21,2 & 12,7 & 2,1 & 2,1 \\
Esc. os cabelos & 98,9 & 1,1 & - & - & - & 91,4 & 5,3 & 2,1 & 1,1 & - \\
Levantar os braços & 97,8 & 1,1 & 1,1 & - & - & 92,5 & 4,2 & 3,2 & - & - \\
Tomar banho & 96,8 & 2,2 & - & - & - & 93,6 & 4,2 & 2,1 & - & - \\
Lavar os cabelos & 97,8 & 2,1 & - & - & - & 93,6 & 3,2 & 3,2 & - & - \\
Vestir camiseta & 96,8 & 2,2 & 1,1 & - & - & 92,5 & 4,2 & 2,1 & 1,1 & - \\
Preparar lanche & 97,8 & 1,1 & 1,1 & - & - & 94,6 & 2,2 & 3,2 & - & - \\
Andar em subidas & 88,3 & 3,2 & 5,3 & 2,1 & 1,1 & 54,2 & 17,0 & 22,3 & 6,3 & - \\
Subir alg. Degraus & 88,3 & 7,4 & 2,2 & 2,2 & - & 63,8 & 22,3 & 10,6 & 3,2 & - \\
Andar poucos m. & 94,6 & 2,2 & 3,1 & - & - & 84,0 & 8,5 & 3,2 & 4,2 & - \\
Andar local acidentado & 90,4 & 5,3 & 1,06 & 3,1 & - & 72,3 & 19,1 & 4,2 & 4,2 & - \\
\hline
\end{tabular}

* Hoje é referente ao dia da entrevista.

Legenda: N: nenhuma / L: leve / M: moderada / G: grave / MG: muito grave 
Tabela 4 - Correlação entre tempo de prática de hidroginástica, medidas antropométricas, dispneia e fadiga, Santa Maria, RS, 2014.

\begin{tabular}{lcccc}
\hline & Dispneia & $\begin{array}{c}\text { Dispneia nas } \\
\text { atividades diárias }\end{array}$ & Fadiga & $\begin{array}{c}\text { Fadiga nas } \\
\text { atividades diárias }\end{array}$ \\
\hline Tempo prática & $-0,18(0,07)$ & $-0,19(0,06)$ & $-0,25\left(0,01^{*}\right)$ & $-0,14(0,16)$ \\
\hline Massa Corporal & $0,20(0.05)$ & $0,22\left(0,02^{*}\right)$ & $0,26\left(0,01^{\star}\right)$ & $0.21\left(0.003^{*}\right)$ \\
IMC $\left(\mathrm{Kg} / \mathrm{m}^{2}\right)$ & $0,25\left(0,01^{*}\right)$ & $0,28\left(0,005^{\star}\right)$ & $0,24\left(0,01^{*}\right)$ & $0.30\left(0.002^{*}\right)$ \\
RCQ $(\mathrm{cm})$ & $0,03(0,71)$ & $0,001(0,98)$ & $0,02(0,83)$ & $0,13(0,21)$ \\
\hline
\end{tabular}

p de Sperman. ${ }^{*} p<0,05$. IMC - Índice de massa corporal. CC - Circunferência da cintura. RCQ - Relação cintura-quadril. Dispneia - falta de ar na maioria dos dias, no dia da entrevista e nas atividades do cotidiano. Fadiga - cansaço muito grande na maioria dos dias, no dia da entrevista e nas atividades do cotidiano.

\section{DISCUSSÃO}

As medidas antropométricas de acordo com o IMC, apontam para pré-obesidade, média de $28 \mathrm{Kg} / \mathrm{m}^{2}$, representando um risco aumentado para morbidades ${ }^{13}$, de igual forma os escores médios de CC $(93,6 \mathrm{~cm})$ e RCQ $(0,87 \mathrm{~cm})$, estão acima do limite ideal, $\geq 80 \mathrm{~cm}$ e $\geq 0,8 \mathrm{~cm}$ respectivamente, representando um aumento substancial no risco de morbidades ${ }^{13,14}$.

Não existe um consenso na literatura quanto aos pontos de corte dos valores tidos como ideais na população idosa, visto que com o envelhecimento, ocorrem alterações na composição corporal dos indivíduos ${ }^{13,14}$. Contudo, principalmente as medidas de CC e RCQ mostram-se relevantes devido ao importante impacto da localização da gordura corporal sobre as doenças cardiovasculares, pois a gordura abdominal é um fator de risco para dislipidemias, hipertensão arterial, resistência à insulina e diabetes, condições determinantes sobre as doenças cardiovasculares, além disso a CC aumentada eleva a probabilidade de utilização de atendimento em prontosocorro $^{15,16}$. Dados mostram que a obesidade é maior entre as mulheres, apresentando maior pico de ganho entre 45 e 64 anos $^{17}$.

Em estudo realizado com adultos, usuários de unidade básica de saúde, encontrou-se o valor da média do IMC, CC e RCQ menores do que o presente estudo, bem como menor proporção de indivíduos que não foram hospitalizados nos últimos doze meses ${ }^{18}$.

Ainda, referente aos fatores de risco para internação hospitalar, estes vêm de encontro os dados de Estrella et al. ${ }^{12}$, realizado com 1.423 idosos de uma operadora de saúde do Rio de Janeiro/BR, no qual destaca-se que $14,5 \%$ foram internados pelo menos uma vez e 33\% tiveram quatro ou mais consultas. Corroborando, Travassos et al. ${ }^{19}$, apontam que as mulheres utilizam os serviços de saúde mais do que os homens.

Nesse contexto, os resultados mostramse mais expressivos, haja vista que a grande maioria $(89,3 \%)$ das idosas não passou por nenhuma internação nos últimos 12 meses e no mesmo período $26,6 \%$ não realizaram nenhuma consulta para tratamento e 26,6\% realizaram apenas uma consulta no mesmo período. O que aponta para os efeitos positivos da prática de exercício físico.

Neste estudo a prevalência de diabetes mellitus II (DM II) foi de 17\%, corroborando a este dado Rosa et al. ${ }^{17}$, apontam para o 
aumento da prevalência de DM II na população idosa, podendo comprometer até cerca de $20 \%$ dos idosos, sendo associada a mortalidade principalmente no sexo feminino.

É importante ressaltar a percepção de saúde do grupo, onde apenas 2,1\% (2 mulheres) consideram sua saúde ruim, sendo que Alves e Rodrigues ${ }^{20}$ ressaltam que à medida que o grau de dependência e presença de doenças crônicas aumentam, maior é a chance de o idoso auto perceber sua saúde ruim.

Por sua vez, fadiga e dispneia são sintomas de diversas doenças cardiovasculares e respiratórias, como a insuficiência cardíaca e a DPOC, com um grande impacto sobre a qualidade de vida das pessoas ${ }^{6,21}$. Segundo o DATASUS ${ }^{22}$ morrem no Brasil três pessoas por hora em decorrência da DPOC, popularmente conhecida como bronquite crônica ou enfisema pulmonar. A doença leva à dificuldade de respirar e ao cansaço progressivo que dificulta a realização de atividades simples do dia a dia como subir escadas, andar, trocar de roupa ou tomar banho. Fatos que tornam de suma importância a investigação destes sintomas, tendo em vista que os mesmos dependem do autorrelato para serem identificados ${ }^{21}$.

Porém, a fadiga é um fenômeno subjetivo e com múltiplas causas que envolvem aspectos físicos, cognitivos e emocionais ${ }^{21}$, portanto a partir da detecção da presença desses sintomas, por parte dos idosos, familiares ou de profissionais da saúde, torna-se imprescindível a busca por diagnóstico para realizar o tratamento adequado.

Por meio do diagnóstico adequado, seja ele para a presença ou não de doenças, o exercício físico é um aliado no tratamento e prevenção, nesse sentido a hidroginástica tem sido importante possibilidade para prática de exercícios físicos, principalmente na população idosa $^{9,10}$. Um estudo realizado com 74 idosos em Recife-BR, onde os mesmos eram sedentários a pelo menos 12 meses, demonstrou que houve melhora na aptidão física ${ }^{23}$. Nakagava e Rabelo ${ }^{24}$ em um estudo com 22 idosas em Timóteo- MG/ $\mathrm{BR}$, encontraram melhora no perfil de qualidade de vida das idosas praticantes de hidroginástica. O mesmo é relato por Assis et al. ${ }^{8}$ afirmando que a hidroginástica na terceira idade melhora o condicionamento físico, a saúde e a autoestima dos idosos.

A hidroginástica mostra-se uma possibilidade, inclusive para idosos com possíveis limitações, possibilitando melhoras significativas na saúde desta população. No presente estudo, o tempo médio de prática de hidroginástica foi de 6,4 anos o que demonstra a adesão a este exercício. Um fator de suma importância, visto que o sedentarismo é um dos grandes responsáveis pela dependência na realização das atividades da vida diária e para a diminuição da capacidade funcional ${ }^{25}$.

Os resultados encontrados apontam os benefícios da hidroginástica perante as variáveis estudadas, visto que mesmo ao apresentarem medidas antropométricas que demonstram um risco substancialmente aumentado de comorbidades cardiovasculares e diabetes, as mesmas foram relatas por apenas $14,8 \%$ e $17,2 \%$, respectivamente, sendo que $89 \%$ não tiveram nenhuma internação nos últimos 12 meses.

O tempo de prática de hidroginástica não apresentou correlações significativas, com o IMC, CC, RCQ, peso e dispneia, contudo apresentou correlação negativa estatisticamente significativa com a fadiga, demonstrando que estas covariam em direções opostas, o que pode estar relacionado aos benefícios da prática de hidroginástica e a própria imersão, devido as suas propriedades da água, como a pressão hidrostática, que podem gerar aumento de força e resistência muscular devido à resistência da água durante o exercício ${ }^{26}$.

O peso, IMC e CC apresentaram correlações positivas estatisticamente significativas com dispneia e fadiga, logo estas covariam na mesma direção, demonstrando que estes indicadores influenciam na percepção do grupo estudado perante estes fatores de risco. Ressaltando que, embora não haja consenso sobre os valores ideais, estes continuam sendo importantes indicadores de saúde.

Tem-se como limitação do estudo a seleção amostral, a qual foi realizada por conveniência, ainda se admite a possível influência de outras morbidades não analisadas e fatores comportamentais, como alimentação e tabagismo. 


\section{CONCLUSÃO}

Apesar das limitações do estudo, em torno de 5\% referiram possui-las em grau consideramos que a partir das condições de grave/muito grave. Porém esses fatores são saúde analisadas, é possível apontar os efeitos subjetivos e acometem indivíduos com alguma positivos às idosas praticantes de hidroginástica patologia, assim como indivíduos saudáveis, em relação a saúde, visto que mesmo com portanto, a partir da detecção desses sintomas resultados antropométricos apontando para é essencial a busca por diagnóstico preciso, risco acentuado de morbidades, a grande para que o tratamento adequado seja seguido. maioria das idosas relataram boas condições Sugere-se que estudos futuros sejam realizados de saúde, baixo número de consultas para visando investigar também as variáveis que tratamento e internações hospitalares.

Quanto a dispneia e fadiga, percentual possam influenciar os resultados, tais como as supracitadas nas limitações do estudo.

\section{REFERÊNCIAS}

1. Instituto Brasileiro de Geografia e Estatística - IBGE. Projeções e estimativas da população do Brasil e das Unidades da Federação. 2013. Disponível em: http://goo.gl/pb23XR.

2. World Health Organization - WHO. Relatório Mundial de Envelhecimento e Saúde. 2015.

3. Cheng et al. Chronic disease mortality in rural and urban residents in Hubei Province, China, 2008-2010. BMC Public Health. 2013; 2(13): 713-721.

4. Góis ALB, Veras RP. Informações sobre a morbidade hospitalar em idosos nas internações do Sistema Único de Saúde do Brasil. Cien Saúde Coletiva. 2010; 15(6): 2859-2869.

5. Brasil. Ministério da Saúde. Cadernos de Atenção Básica: Doenças Respiratórias Crônicas. Brasília-DF, 2010.

6. Santos ML, Rosa RJ, Mattagin AM. Avaliação da capacidade funcional, humor e cognição em pacientes portadores de DPOC. ConSientiae Saúde, São Paulo. 2009; 8(2): 267-273.

7. Luiz AF, Oliveira GF. Estudo da força muscular respiratória em idosos institucionalizados de Foz do Iguaçu. Il Seminário de Fisioterapia da UNIAMERICA; 2008. Foz do Iguaçu, PR; 2008.

8. Assis RS, Silva Junior LFS, Santos LR, Navarro AC. A hidroginástica melhora o condicionamento físico dos idosos. Revista Brasileira de Prescrição e Fisiologia do Exercício, São Paulo. 2007; 1(5): 62-75.

9. Kruel LFM. Alterações fisiológicas e biomecânicas em indivíduos praticando exercícios de hidroginástica dentro e fora da água. [Tese]. Santa Maria: Ciências do Movimento Humano, Universidade Federal de Santa Maria; 2000.

10. Albuquerque IM, Emmanouilidis A, Ortolan T, Cardoso DM, Gass R, Jost RT, et al. Capacidade funcional submáxima e força muscular respiratória entre idosas praticantes de hidroginástica e dança: um estudo comparativo. Revista Brasileira de Geriatria e Gerontologia, Rio de Janeiro. 2013; 16(2): 327-336.

11. Kolevis D, Segretti NO, Probst S, Lareau SC, Brunetto AF, Pitta F. Validação do Modified Pulmonary Functional Status and Dyspnea Questionnaire e da escala do Medical Research Council para o uso em pacientes com doença pulmonar obstrutiva crônica no Brasil. Jornal Brasileiro de Pneumolologia. 2008; 34(12): 1008-1018.

12. Estrella K, Oliveira CEF, Sant'Anna AA, Caldas CP. Detecção do risco para internação hospitalar em população idosa: um estudo a partir da porta de entrada no sistema de saúde suplementar. Cadernos de Saúde Pública, Rio de Janeiro. 2009; 25(3): 507-512.

13. Godoy-Matos AF, Oliveira J, Guedes EP, Carraro L, Lopes AC, Mancini MC, et al. (part.). Diretrizes Brasileiras de Obesidade ABESO. Associação Brasileira para estudo da obesidade e da síndrome metabólica. 3.ed. - Itapevi, SP. AC Farmacêutica, 2009.

14. Lópes-Jaramillo P, Sánchez RA, Diaz M, Cobos L, Bryce A, Parra-Carrillo JZ, et al. Consenso latino-americano de hipertensão em pacientes com diabetes tipo 2 e síndrome metabólica. Arq Bras Endocrinol Metab. 2014; 58(3).

15. Tomasi E, Nunes BP, Thumé E, Silveira DS, Siqueira FV, Piccini RX, et al. Utilização de serviços de saúde no Brasil: associação com indicadores de excesso de peso e gordura abdominal. Cad Saúde Pública 2014; 30(7): 1515-1524.

16. Pimenta IL, Sanches RC, Pereira JPR, Houri BF, Machado ELG; Machado FSG. Medidas das circunferências abdominal e cervical para mensurar riscos cardiovasculares. Rev Méd Minas Gerais, 2014; 24(S9): S16-19.

17. Rosa C, Brunelli AV, Seibel R, Sturmer J, Silva BA. Aspectos Nutricionais do Envelhecimento. In: Garces SBB, Brunelli AV, Rosa CB (Coord.) O envelhecimento na (Pós) Modernidade: Uma visão Interdisciplinar. ljuí; Editora Unijuí, 2012, p. 13-29.

18. Ramalho JRO, Lopes ACS, Toledo MTT, Peixoto SWV. Nível de atividade física e fatores associados ao sedentarismo em usuários de uma unidade básica de saúde em Belo Horizonte, Minas Gerais. Rev Mineira de Enfermagem. 2014; 18(2): 426-432.

19. Travassos C, Viacava F, Pinheiro R, Brito A. Utilização dos serviços de saúde no Brasil: gênero, características familiares e condição social. Rev Panam Salud Publica. 2002; 11(5-6), 365-73.

20. Alves LC, Rodrigues RN. Determinantes da auto-percepção de saúde entre idosos do município de São Paulo, Brasil. Rev Panam Salud Publica. 2005; 17(5/6): 331-341.

21. Fini A, Cruz DALM. Características da fadiga de pacientes com insuficiência cardíaca: revisão de literatura. Rev Latino-Americana de Enfermagem. 2009; 17(4). 
22. Brasil. Ministério da Saúde. Departamento de Informática do SUS (Datasus). Informações de saúde. Acesso em: 2 dez. 2014. Disponível em: http://datasus.saude.gov.br/nucleos-regionais/alagoas/noticias-alagoas/565-bronquite-cronica-causa-40-mil-mortes-acada-ano-revela-dados-do-datasus.

23. Alves RV, Mota J, Costa MDC, Alves JGB. Aptidão física relacionada à saúde de idosos: influência da hidroginástica. Revista Brasileira de Medicina do Esporte. 2004; 10(1), 31-37.

24. Nakagava BKC, Rabelo RJ. Perfil da qualidade de vida de mulheres idosas praticantes de hidroginástica. MOVIMENTUM - Revista Digital de Educação Física - Ipatinga: Unileste-MG. 2007; 2(1).

25. Krug MR, Garces SBB, Romiti JC. Nível de atividade física e capacidade funcional de idosos. In: Garces SBB, Brunelli AV, Rosa CB (Coord.) O envelhecimento na (Pós) Modernidade: Uma visão Interdisciplinar. ljuí; Editora Unijuí, 2012. p. 79-100.

26. Silva RS; Nascimento Júnior JRA; Vieira LF; Oliveira DV. Qualidade de vida e capacidade funcional de idosas praticantes de hidroginástica no município de Sarandi/PR. Rev Bras Qual Vida. 2016; 8(1): 28-41. 